\title{
39-jähriger Patient mit Aspirations-Pneumonie bei hypomotiler Achalasie
}

\author{
39-Year-Old Patient Presenting with Aspiration Pneumonia \\ Due to High Hypomotile Achalasia
}

Autoren

Institut

\author{
S. Ewig', M. Bollow ${ }^{2}$, U. Henkelüdecke ${ }^{3}$
}

Thoraxzentrum Ruhrgebiet, Standort Bochum, Augusta-Kranken-Anstalt Bochum

Institut für Radiologie, Nuklearmedizin und Radioonkologie, Augusta-Kranken-Anstalt Bochum

Klinik für Allgemeine Innere Medizin und Gastroenterologie, Augusta-Kranken-Anstalt Bochum
Bibliografie

DOI $10.1055 / \mathrm{s}-2006-932222$

Pneumologie 2006; 60;

472-473

(c) Georg Thieme Verlag KG

Stuttgart $\cdot$ New York

ISSN 0934-8387

Korrespondenzadresse Prof. Dr. med. Santiago Ewig Thoraxzentrum Ruhrgebiet, Standort Bochum AugustaKranken-Anstalt Bochum Bergstraße 26 44791 Bochum ewig@augusta-bochum.de
Ein 39-jähriger, bisher gesunder Patient wurde mit Fieber und Dyspnoe stationär eingewiesen. In der p.a.-Röntgen-Thorax-Aufnahme zeigte sich im rechten Mittel- und Unterfeld ein ausgedehntes Infiltrat mit positivem Pneumobronchogramm sowie einem Pleuraerguss mit Kompressionsdystelektasen. Rechts paratracheal fiel eine dorsal gelegene unklare konvex geformte Struktur mit linearer Berandung auf (Abb. 1). Unter der Arbeitsdiagnose einer ambulant erworbenen Pneumonie erhielt der Patient zunächst eine antimikrobielle Therapie mit Amoxicillin/ Clavulansäure in Form einer Sequenztherapie. In der zur weiteren Abklärung durchgeführten Mehrzeilen-CT des Thorax bestätigte sich in den ventralen Schichten der koronaren Rekonstruktion der Befund eines paratrachealen Substrates mit Flüssigkeits- und Gaseinschlüssen (Abb. 2a). In den dorsalen koronaren Schichten (Abb. 2c) und in einer sagittalen Rekonstruktion (Abb. 2b) zeigte sich ein wandverdickter Mega-Ösophagus mit reichlich Succus. Sagittal (Abb. 2b) und transversal (Abb. 2a) Darstellung einer Luft-Succus-Spiegelbildung, welche bis zum Niveau des oberen Ösophagussphinkters heranreichte. In einer Breischluckuntersuchung zeigte sich ein Kontrastmittelstop am ösophagogastralen Übergang mit einer typischen Weinglaskonfiguration, der nahezu beweisend für das Vorliegen einer Achalasie (Abb. 3a) war. Die Gastroskopie bestätigte diesen Verdacht. Eine 60 Minuten später durchgeführte Übersichtsaufnahme im Liegen (Abb. 3b) zeigte das Persistieren des jodhaltigen Röntgenkontrastmittels

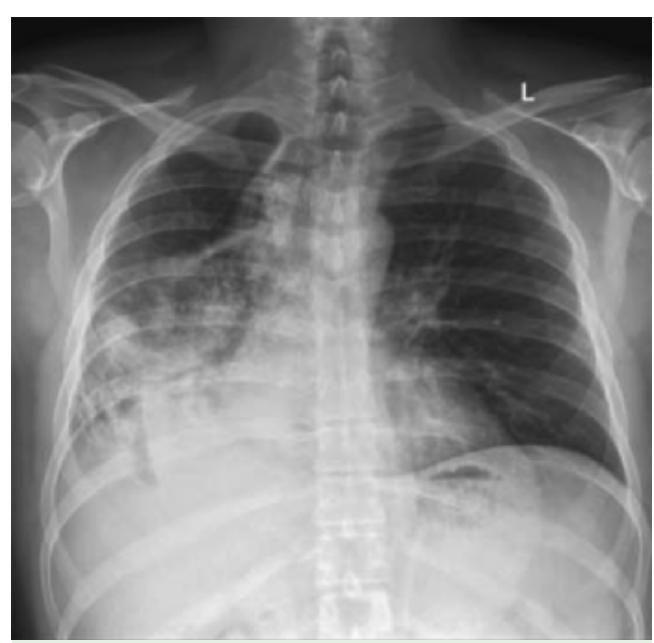

Abb. 1 Pneumologisches Infiltrat rechts, unklare konvex geformte Struktur rechts paratracheal dorsal

im Ösophagus als Ausdruck des kompletten Kontrastmittelstops. In einer Öesophagusdarstellung nach mehreren Bougierungsbehandlungen (Abb. 4) war eine Wiederherstellung einer ösophagogastrischen Passage durch den unteren Ösophagussphinkter darstellbar.

Erst nachträglich konnte eruiert werden, dass seit langem Regurgitationen bestanden. Die Gastroskopie bestätigte den Befund einer hypomotilen Achalasie.

Auf diesem Hintergrund musste die Pneumonie als Aspirations-Pneumonie angesehen werden. Diese konnte erfolgreich behandelt werden. 

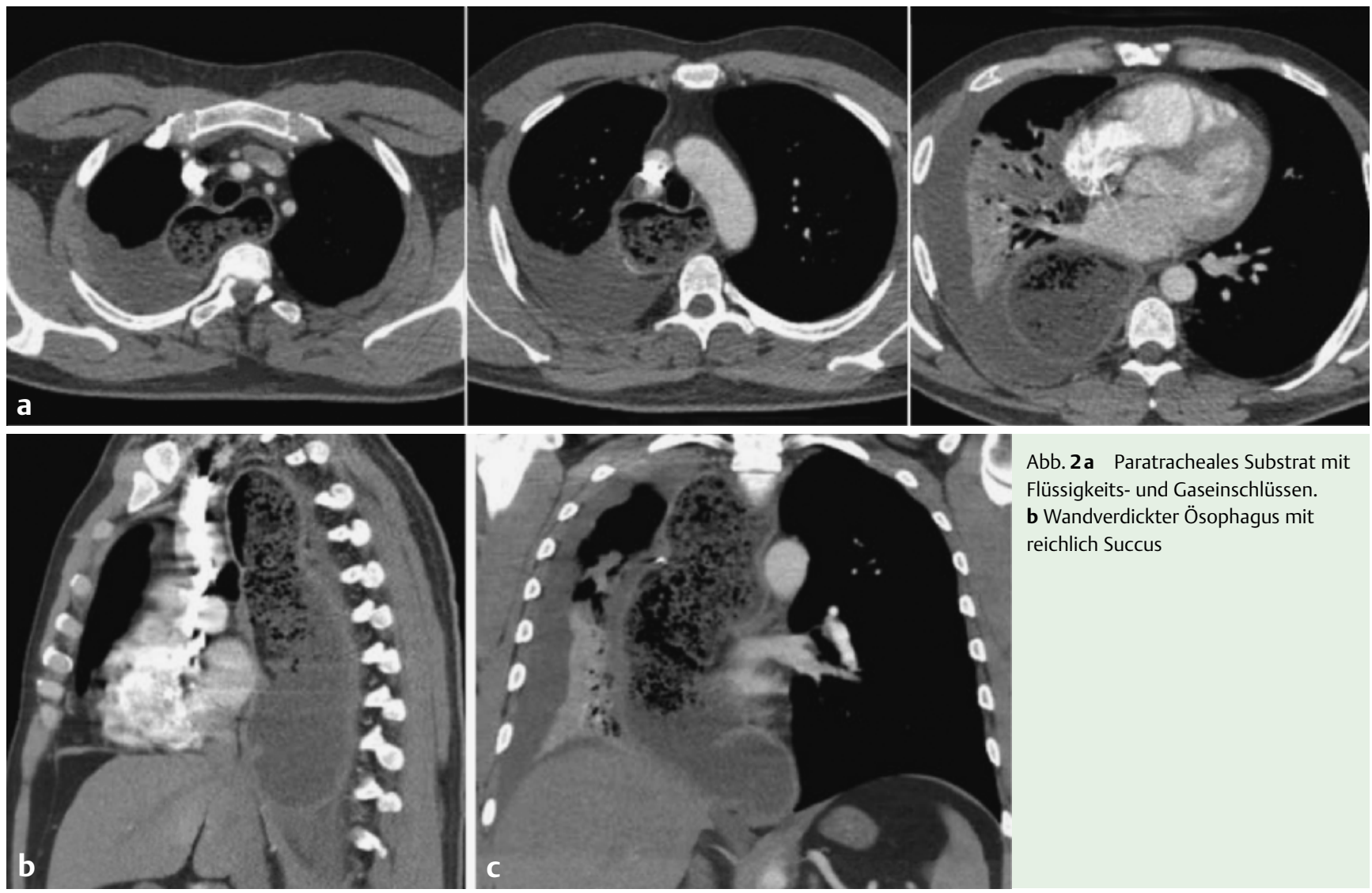

Abb. 2 a Paratracheales Substrat mit

Flüssigkeits- und Gaseinschlüssen.

b Wandverdickter Ösophagus mit

reichlich Succus
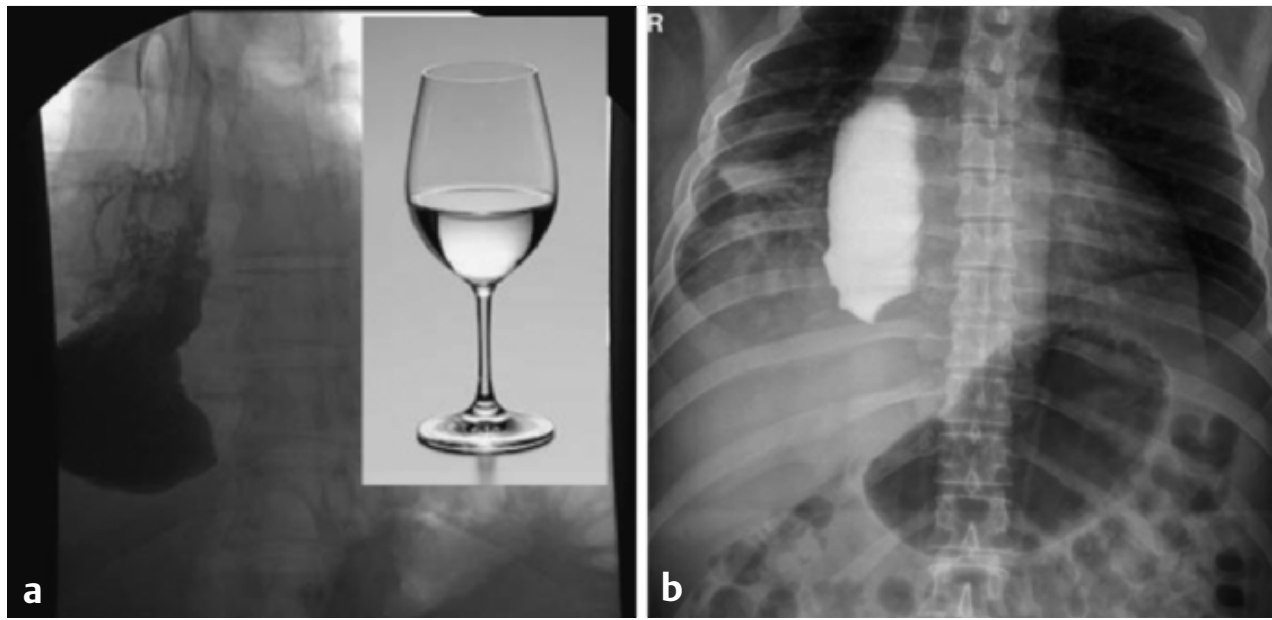

Abb. 3a KM-Darstellung des

ösophago-gastralen Übergangs:

typische Weinglaskonfiguration bei

Achalasie

b Spätaufnahme nach 60 Minuten:

Persistieren des Kontrastmittels

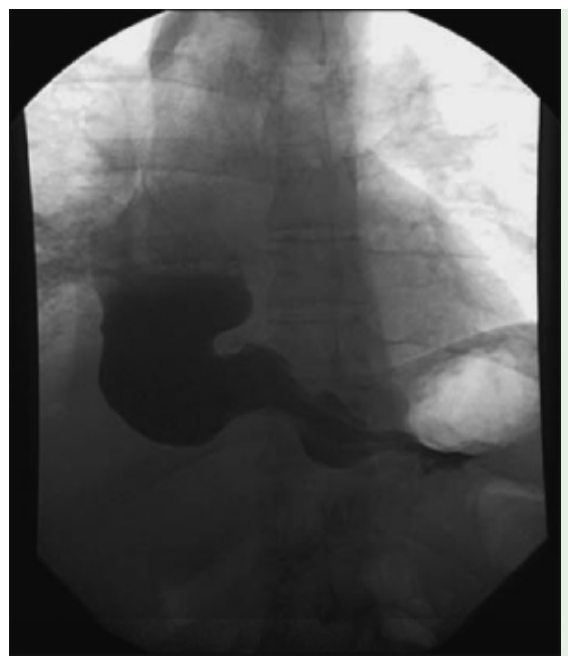

Abb. 4 Z.n. mehrfach

Bougierungsbehand-

lungen: Widerherstel-

lung der ösophago-

gastrischen Passage 